\title{
A SYSTEM OF MUTUALLY CONTRADICTORY $n$ ABSTRACTIONS WHOSE PROPER SUB-SYSTEMS ARE ALL MUTUALLY CONSISTENT
}

\author{
KATUZI ONO and MINOLU OHTA
}

It has been pointed out by $\mathrm{K}$. ONO ${ }^{1)}$ that there is a pair of mutually contradictory abstractions, each of which is self-consistent. Afterwards, Y. INOUE $^{2)}$ pointed out that there is a vast class of such pairs which is as vast as the class of all the Russell-type paradoxes. It must be a natural course of matter to ask the following question: For every number $n$, is there a system of mutually contradictory abstractions whose proper sub-systems are all mutually consistent?

In the present paper, we would like to show that for any number $n$, we can really give a system of $n$ such abstractions that they contradict mutually as a whole but any proper sub-system of it is proved to be mutually consistent and further all the abstractions in the system except one are stratified $^{3)}$. In so far as Quine's $\mathrm{NF}^{4}$ ) is consistent, there is no mutually contradictory system of $n$ abstractions which are all stratified.

The following lemma can be proved easily.

Lemma. $\quad(\exists p)(x) x \in p \rightarrow \mathfrak{F} \quad$ implies

$$
\mathfrak{F} \equiv(\exists p)(x)(x \in p \equiv(\mathfrak{F} \rightarrow x \in m)),
$$

and the proposition

$$
\mathfrak{F} \equiv(\exists p)(x)(x \in p \equiv((\mathfrak{F} \rightarrow x \in m) \wedge(x \in x \rightarrow \mathfrak{F})))
$$

holds tautologically. ${ }^{5}$

Received August 28, 1967.

1) See K. Ono [2].

2) See $Y$. Inoue [1].

3),4) As for "stratified" and NF, see W.V.O. Quine [3], also [4].

5) We have spoken on the subject of the present paper on May 15, 1967 at the 1967 Annual Meeting of Math. Soc. Jap. At that time, we have shown another example of a system of mutually contradictory $n$ unstratified abstractions whose proper sub-systems are all consistent. The example system has been given by the following lemma:

$$
\mathfrak{F} \rightarrow(\exists p)(x) x \notin p \quad \text { implies } \mathfrak{F} \equiv(\exists p)(x)(x \in p \equiv(\neg \mathfrak{F} \wedge x \notin x)) \text {. }
$$

M. Ohta has spoken on the lemma at the same meeting in his "Presentation of set theory by a single abstraction axiom (Japanese)". The example system given in the present paper seems a little simpler and more transparent, and moreover, we can take all the propositions but one in the system as stratified. 
Now, for any number $n[2 \leq n]$, we prove that the system of $n$ abstractions

$$
\begin{aligned}
& \mathscr{S}_{i} \equiv(\exists p)(x)\left(x \in p \equiv\left(\mathfrak{F}_{i} \rightarrow x \in m\right)\right) \quad[i=1, \cdots, n-1], \\
& \mathscr{S S}_{n} \equiv(\exists p)(x)\left(x \in p \equiv\left(\left(\mathfrak{F}_{n} \rightarrow x \in m\right) \wedge\left(x \in x \rightarrow \mathfrak{F}_{n}\right)\right),\right.
\end{aligned}
$$

for any free parameter $m$, are mutually contradictory but all the proper sub-systems of it are consistent, and moreover, $\mathscr{S}_{1}, \cdots, \mathbb{S}_{n-1}$ are all stratified, where

$$
\begin{aligned}
\mathfrak{U} & \equiv(\exists p)(x) x \in p, \\
\mathfrak{B}_{i} & \equiv\left(\exists x_{0}\right) \cdots\left(\exists x_{i}\right)\left(x_{0} \in x_{1} \wedge \cdots \wedge x_{i-1} \in x_{i}\right), \\
\mathfrak{F}_{1} & \equiv\left(\mathfrak{A} \vee \mathfrak{B}_{1}\right), \\
\mathfrak{F}_{i} & \equiv\left(\mathfrak{A} \vee\left(\mathfrak{B}_{i-1} \rightarrow \mathfrak{B}_{i}\right)\right) \quad[1<i<n], \\
\mathfrak{F}_{n} & \equiv\left(\neg \mathfrak{A} \wedge \rightarrow \mathfrak{B}_{n-1}\right) .
\end{aligned}
$$

At first, we remark that $\mathbb{S}_{\imath}$ is equivalent to $\mathfrak{F}_{i}$ for any $i[1 \leq i \leq n]$. For,

$$
(\exists p)(x) x \in p \rightarrow \mathfrak{F}_{i}, \quad \text { i.e., }, \quad \mathfrak{A} \rightarrow \mathfrak{F}_{i}
$$

holds trivially for any $i$ less than $n$ by definition. Accordingly, the abstraction

$$
\mathbb{S}_{i}, \quad \text { i.e., } \quad(\exists p)(x)\left(x \in p \equiv\left(\mathfrak{\mho}_{i} \rightarrow x \in m\right)\right)
$$

is equivalent to $\mathfrak{F}_{i}$ by the first half of the lemma. On the other hand, the abstraction

$$
\mathbb{S}_{n}, \quad \text { i.e., } \quad(\exists p)(x)\left(x \in p \equiv\left(\left(\mathfrak{F}_{n} \rightarrow x \in m\right) \wedge\left(x \in x \rightarrow \mathfrak{F}_{n}\right)\right)\right)
$$

is equivalent to $\mathfrak{F}_{n}$ by the latter half of the lemma. Accordingly, to prove that the system of abstractions $\mathscr{S}_{1}, \cdots, \mathscr{S}_{n}$ are mutually contradictory, we have only to prove that the system of propositions $\mathfrak{F}_{1}, \cdots, \mathfrak{F}_{n}$ are mutually contradictory. Also, to prove that all the proper sub-systems of the system of abstractions $\mathscr{S}_{1}, \cdots, \mathscr{S}_{n}$ are consistent, we have only to prove that all the proper sub-systems of the system of propositions $\mathfrak{F}_{1}, \cdots, \mathfrak{F}_{n}$ are consistent.

Secondly, we can see easily by definition that the abstractions $\mathfrak{S}_{1}$, $\cdots, \otimes_{n-1}$ are surely stratified.

Thirdly, we prove that the propositions $\mathfrak{F}_{1}, \cdots, \mathfrak{F}_{n}$ are mutually contradictory. To show this, let us assume $\mathfrak{F}_{1}, \cdots, \mathfrak{F}_{n}$. Then, $\rightarrow \mathfrak{A}$ holds, because $\mathfrak{F}_{n}$ implies it. Hence, $\mathfrak{F}_{1}$ is equivalent to $\mathfrak{B}_{1}$, and $\mathfrak{F}_{i}$ is equivalent 
to $\mathfrak{B}_{i-1} \rightarrow \mathfrak{B}_{i}$ for $1<i<n$. Accordingly, $\mathfrak{B}_{n-1}$ is deducible from $\mathfrak{F}_{1}, \cdots, \mathfrak{F}_{n}$. On the other hand, $\rightarrow \mathfrak{B}_{n-1}$ is decucible from $\mathfrak{F}_{n}$. Hence, the system of propositions $\mathfrak{F}_{1}, \cdots, \mathfrak{F}_{n}$ are mutually contradictory.

Lastly, we will prove that any proper sub-system $S$ of the system of propositions $\mathfrak{F}_{1}, \cdots, \mathfrak{F}_{n}$ are mutually consistent. To show this, let us assume that $\boldsymbol{S}$ does not contain $\mathfrak{F}_{i}[1 \leq i \leq n]$.

Now, in the model domain $\{1, \cdots, i\}$ with the binary relation " $x \in y$ " which is defined as standing for " $x$ is a natural number less than the natural number $y$ ", the proposition $\rightarrow \mathfrak{A}$ holds. The proposition $\mathfrak{B}_{k}$ holds for every $k$ less than $i$, but it does not hold for every $k$ equal to or bigger than $i$. Accordingly, the proposition $\mathfrak{F}_{1}$ holds unless $i$ is 1 , every proposition $\mathfrak{F}_{k}$ for $1<k<n$ holds except for the case $k=i$, and the proposition $\mathfrak{F}_{n}$ holds unless $i$ is $n$. Anyway, all the propositions $\mathfrak{\mho}_{k}[1 \leq k \leq n]$ holds true except for the case $k=i$. Because the system $\boldsymbol{S}$ of propositions does not contain $\mathfrak{F}_{i}$, all the propositions of $\boldsymbol{S}$ hold in the model domain $\{1, \cdots, i\}$. Hence, $\boldsymbol{S}$ is consistenst.

\section{REFERENCES}

[1] Inoue, Yoshinobu: On systems of self-consistent abstractions, Nagoya Math. J., 28 (1966), 179-185.

[2] Ono, Katuzi: Mutual contradiction of two self-consistent abstractions, Nagoya Math. J., 28 (1966), 59-61.

[3] Quine, Willard Van Orman: Set theory and its logic. Cambridge, Mass., xv+359.

[4] - New foundation for mathematical logic, Amer. Math. Monthly, 44 (1937), 70-80.

\section{Mathematical Institute,}

Nagoya University.

Added in proof May 8, 1968.

The definition of $\mathfrak{F}_{i}[1 \leq i \leq n]$ in p. 26 can be simplified as follows:

$$
\begin{aligned}
& \mathfrak{F}_{1} \equiv \mathfrak{B}_{1}, \\
& \mathfrak{F}_{i} \equiv\left(\mathfrak{B}_{i-1} \rightarrow \mathfrak{B}_{i}\right) \quad[1<i<n], \\
& \mathfrak{F}_{n} \equiv \rightarrow \mathfrak{B}_{n-1} .
\end{aligned}
$$

For, one can prove $\mathfrak{A} \rightarrow \mathfrak{B}_{i}$ by definition. 